\title{
UNE ANALYSE DE LA RÉGULATION DES COMMUNS SCIENTIFIQUES À PARTIR DES CONVENTIONS ET DES IMAGINAIRES LINGUISTIQUES
}

\author{
Claude Paraponaris, Anne Rohr
}

De Boeck Supérieur | «Innovations »

2020/3 N 63 | pages 39 à 67

ISSN 1267-4982

ISBN 9782807394209

Article disponible en ligne à l'adresse :

https://www.cairn.info/revue-innovations-2020-3-page-39.htm

Distribution électronique Cairn.info pour De Boeck Supérieur.

(C) De Boeck Supérieur. Tous droits réservés pour tous pays.

La reproduction ou représentation de cet article, notamment par photocopie, n'est autorisée que dans les limites des conditions générales d'utilisation du site ou, le cas échéant, des conditions générales de la licence souscrite par votre établissement. Toute autre reproduction ou représentation, en tout ou partie, sous quelque forme et de quelque manière que ce soit, est interdite sauf accord préalable et écrit de l'éditeur, en dehors des cas prévus par la législation en vigueur en France. Il est précisé que son stockage dans une base de données est également interdit. 


\title{
Une analyse de la régulation des communs scientifiques à partir des conventions et des imaginaires linguistiques
}

\author{
Claude PARAPONARIS \\ Aix Marseille Université \\ Centre National de la Recherche Scientifique \\ Laboratoire d'Économie et de Sociologie du Travail, UMR 7317 \\ claude.paraponaris@univ-amu.fr
}

\begin{abstract}
Anne ROHR
Université Toulouse 2 - Jean Jaurès Éducation, Formation, Travail, Savoirs, UMR 5263

anne.rohr@univ-tlse2.fr
\end{abstract}

\section{RÉSUMÉ}

Notre article propose une analyse typologique de la régulation des communs scientifiques à partir de leur activité de codification linguistique. Nous analysons les communs sous l'angle du fonctionnement affiché des communautés animées par leurs membres. Nous interrogeons l'apparente homogénéité des communautés épistémiques qui soutiennent ces communs en opérant une analyse fondée sur les imaginaires linguistiques. Cette interrogation aboutit à une remise en cause de l'homogénéité de la forme " communauté ». Nous procédons, d'une part, au moyen d'une large revue de littérature en sciences sociales, d'autre part, en conduisant une analyse sociolinguistique des énoncés lexicaux issus des autoprésentations de 23 communs. Cette analyse critique aboutit à segmenter l'ensemble des groupes qui élaborent ces communs en deux familles : les communautés au profil stable et régulier et à visée scientifique ou technique, et les collectifs au profil foisonnant et instable et à visée de transformation sociale.

MOTS CLÉS : communs scientifiques, communautés épistémiques, collectifs, imaginaire, linguistique

CODES JEL : D83, O32, Z13 


\section{ABSTRACT \\ Analyzing the Regulation of the Scientific Creative Commons from a Conventional and Imaginary Linguistic Point of View}

We aim to propose a typological analysis of the regulation of scientific creative commons from the linguistic codification activity. We analyze the commons since the point of view of the communities' work. We point the apparent homogeneity of the epistemic communities that support these commons. This leads to a questioning about the homogeneity of the "community". We proceed, on the one hand, by means of a comparison between different research programs developed in the sociology of science and politics, on the other hand, by conducting a sociolinguistic analysis of the lexical statements resulting from the self-presentations of 23 creative commons. This critical analysis leads to segmenting all the groups that develop these commons into two families: communities with a stable and regular profile and scientific aims, and collectives with an abundant and unstable profile and with a view to social transformation.

KEYWORDS: Scientific Commons, Epistemic Communities, Collectives, Imaginary, Linguistic

JEL CODES: D83, O32, Z13

L'univers des communs scientifiques peut faire l'objet de plusieurs analyses. La question de la coordination au sein des groupes de pairs est sans doute celle qui se prête à de nombreuses reformulations depuis la parution de The New Production of Knowledge en 1994 (Gibbons et al., 1994).

Le développement d'Internet a depuis contribué à élargir les frontières de l'activité scientifique. Internet accroît les possibilités de satisfaction des chercheurs académiques (satisfaire la curiosité et accéder à la reconnaissance par les pairs selon Merton, 1973). Mais Internet permet également l'essor d'une économie de la connaissance (Foray, 2004) supportée par des arrangements organisationnels et des hybridations. Cet élargissement des frontières incite à la discussion à propos des modes d'existence des groupes humains qui développent la connaissance (Dang Nguyen et al., 2017). Nous avançons que le terme de communauté est trop englobant et force la compréhension des logiques d'action qui sont à l'œuvre. Selon nous, il n'existe pas une seule forme d'organisation des communs scientifiques, mais plusieurs.

Notre recherche considère les communs suivant la définition établie par Coriat (2015) à partir des travaux fondateurs d'Ostrom (1990) et de Stallman (2002). Un commun rassemble trois existences particulières : une ressource (matérielle ou informationnelle), un mode d'accès à la ressource et des règles 
de partage (droits et obligations des commoners sur la ressource), et une gouvernance particulière de la ressource. Nous étudions uniquement ici des communs informationnels, et ceci sous l'angle spécifique de la gouvernance de la ressource. Notre problématique s'inscrit dans l'analyse des modes de fonctionnement des groupes qui supportent l'élaboration des communs scientifiques, et qui assurent donc la gouvernance de la ressource informationnelle et cognitive. Nous emploierons le terme de " groupe » afin de laisser ouverte sa qualification sous la forme de " communauté » ou de « collectif » qui présentent chacune des modalités particulières de fonctionnement. Notre question de recherche porte sur le rôle des conventions linguistiques dans la coordination des membres d'un groupe (communauté ou collectif). Nous partons du constat de Livet (1994) suivant lequel la compréhension mutuelle n'est jamais certaine et la cohésion des actions toujours incertaine. Faciliter la coordination au sein d'un groupe consiste à élaborer des conventions d'intercompréhension. L'étude des conventions se prête à de multiples développements. Nous les étudions sous l'angle des énoncés lexicaux définis au sein d'un groupe et présentés au public afin de recruter de nouveaux membres ou bien d'élargir son audience.

Notre objectif consiste à questionner l'homogénéité de la catégorie « communauté » pour décrire les groupes qui élaborent des connaissances : quels rapports entretiennent-ils avec la connaissance ? Et quels sont les rôles qu'ils entendent tenir dans la société à partir de cette production de connaissances ? Le plus souvent, c'est une distinction entre communauté de pratique et communauté épistémique qui est posée. Mais le terme de collectif fait régulièrement son apparition (notamment dans la sociologie des sciences) en alternance avec le terme de communauté sans que l'on sache définir les propriétés respectives de ces différents types de groupe. Nous soutenons qu'il existe des différences importantes entre des communautés académiques plutôt stables et engagées dans le développement de leurs capacités, et d'autres groupes à longévité plus réduite et plutôt engagés dans des programmes de transformation sociale. Nous mobilisons un ensemble de cas de communautés académiques ou d'associations scientifiques de manière exploratoire.

Nous discutons dans un premier temps des apports de différents courants des sciences sociales à l'analyse de l'action collective au sein des communautés ou collectifs. L'implication des membres et leur accès à la ressource cognitive sont abordés au travers du langage utilisé (communauté linguistique, Girin, 1990), en particulier au moyen de sa dimension imaginaire (Houdebine, 1993). Après avoir précisé notre méthodologie et présenté les communs que nous avons étudiés, nous établissons les éléments de comparaison entre 
communauté et collectif. Enfin, nous discutons des apports de ce type d'analyse linguistique en termes de dynamique des communs scientifiques.

\section{Des « communautés épistémiques " aux collectifs}

Un vif intérêt a été porté aux communautés en tant que modalité de coordination et d'élaboration de connaissances (David, Foray, 2002). Ces travaux méritent d'être interrogés en termes de pratiques de coordination. Il est intéressant de les mettre en perspective avec d'autres approches issues de la psychologie de l'activité, de la communication et de la linguistique (figure 1). Finalement : quelles sont les règles qui président à l'action collective au sein de ces communs scientifiques?

\section{Des modalités de socialisation pour faciliter la production de connaissances}

Les théories de la socialisation empruntent beaucoup à la sociologie afin de mettre en lumière le lien consubstantiel entre interaction sociale et apprentissage (Brown, Duguid, 1991), puis entre interaction sociale et élaboration de connaissance (Wenger, 1998). Une communauté de connaissance est définie comme un groupe dont les membres s'engagent volontairement dans l'élaboration et le partage de ressources et de connaissances. La pérennité de la communauté se construit au fil du temps sous les traits d'une identité commune. Et la régulation se réalise par la reconduction de normes sociales particulières (Cohendet et al., 2008). Une distinction est établie entre communautés de pratique et communautés épistémiques (Cohendet, Llerena, 2003). Alors que les premières rassemblent des acteurs homogènes autour de pratiques souvent professionnelles, les secondes réunissent des acteurs plus hétérogènes selon une autorité procédurale qui leur permet de normaliser la connaissance sous forme de savoir à des fins de circulation et de reconnaissance. L'autorité procédurale et le slack créatif jouent des rôles de régulation (Cowan et al., 2000). L'autorité procédurale est un ensemble de règles et de procédures plus ou moins explicites auxquelles se soumettent les membres d'une communauté. Le slack créatif est distribué en partie dans la base de connaissance formalisée des organisations non académiques et en partie dans le fonctionnement des communautés académiques.

Lassociation de ces deux notions nous montre qu'il existe plusieurs types de communautés en fonction du degré d'homogénéité des acteurs, elle nous 
montre les alliances possibles entre agir communautaire et organisations tierces. Mais ce courant d'étude a tendance à considérer des communautés stabilisées sans beaucoup disserter ni sur leur émergence, ni sur l'ensemble des modalités de communication interne (Soulier, 2017).

Un autre courant des théories de la socialisation met en évidence le rôle des croyances communes pour mobiliser des acteurs hétérogènes. Dans le champ de la sociologie du politique, on s'intéresse aux communautés épistémiques (Meyer, Molyneux-Hodgson, 2011).

\section{Figure 1 - Les différents champs d'analyse mobilisés pour l'étude des communs scientifiques}

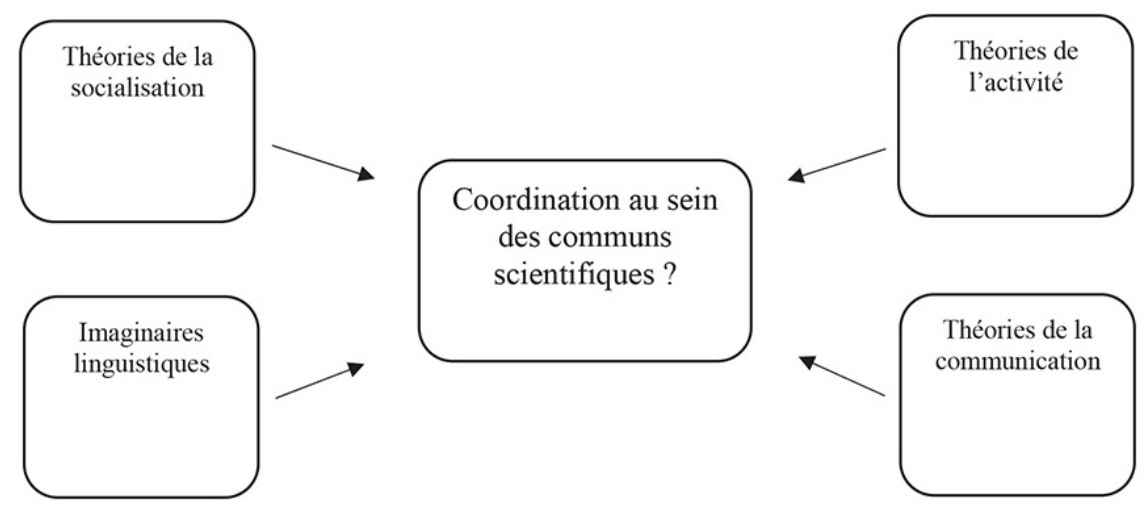

Le terme epistemic community est utilisé par Holzner et Marx (1979) pour rendre compte des communautés qui s'occupaient de politiques environnementales, sanitaires, industrielles ou militaires. Haas (1992) a mis en évidence le rôle des principes et valeurs de l'action sociale. Pour l'auteur, une communauté épistémique désigne : "un réseau de professionnels ayant une expertise et une compétence reconnues dans un domaine particulier et une revendication d'autorité en ce qui concerne les connaissances pertinentes pour les politiques" (Haas, 1992, p. 3). Nous ne sommes plus dans la perspective des communautés épistémiques qui créent et codifient de nouvelles connaissances dans une visée scientifique. Avec ces communautés composées d'experts et de militants, s'ajoute une dimension politique, voire une visée transformatrice. Haas caractérise ces communautés épistémiques par leurs croyances communes et par des principes leur permettant de justifier leurs actions. On voit ainsi apparaitre une distinction entre deux usages de la production des communautés : les premières considèrent les résultats scientifiques comme un moteur de leur activité, alors que les secondes entendent mobiliser en partie ces résultats à des fins extra-académiques. 


\section{Les collectifs scientifiques comme analyse alternative}

Un troisième courant de la socialisation introduit des nuances importantes dans l'approche de ces groupes. La sociologie des sciences considère les fondements des communautés sous l'angle des collectifs scientifiques (Granjou, Peerbaye, 2011). Héritier de travaux fondateurs (Hagstrom, 1965 ; Merton, 1973 ; Bourdieu, 1975), ce champ de recherche interroge les modes d'existence des communautés. Il a inspiré directement notre partition des groupes soutenant les communs scientifiques (distinction entre communautés et collectifs).

Latour (2005) fait remarquer l'hétérogénéité des approches relatives aux communautés, groupes et collectifs scientifiques. Il reprend un auteur oublié, Fleck (1934), pour mettre en lumière l'effet d'entraînement de l'idéologie dans la conduite des activités scientifiques collectives.

Knorr-Cetina (1982) remet en cause le concept de communauté comme modèle de l'organisation scientifique. L'auteure insiste sur la rareté des travaux empiriques et sur le caractère parfois très affirmatif qui est utilisé pour caractériser le fonctionnement communautaire. Elle met en évidence des arènes transépistémiques, qui tout en cadrant l'action, autorisent un grand nombre d'agencements aux issues souvent incertaines. Ces arènes présentent une capacité à façonner le contenu et la forme des projets de recherche, autrement dit l'organisation du travail et la production de connaissances. Latour (2005) insiste sur l'importance des dimensions processuelles. Un collectif ne serait pas un simple préalable à l'activité scientifique. Le collectif émergerait en interaction avec la production des premiers énoncés scientifiques et la circulation des objets techniques. Meyer et Molyneux-Hodgson (2011, p. 149) concluent qu'« au lieu de supposer que les communautés épistémiques existent a priori, nous nous devons d'examiner comment elles naissent, comment elles sont fabriquées et matérialisées, ce qui nécessite l'exploration des pratiques, des objets, des métaphores, des instruments et des discours qui produisent et maintiennent ces collectivités».

Ce troisième courant apporte des éléments complémentaires à l'étude des groupes qui animent les communs scientifiques. D'une part, la confirmation de la rareté des travaux empiriques nous prive d'arguments quant à l'homogénéité ou à la diversité des modes de coordination de ces groupes ; d'autre part, ces travaux nous incitent à remettre en cause l'hypothèse d'une identité commune des membres d'un groupe scientifique. Enfin, l'existence d'une diversité sémantique qui vient questionner notre propre connaissance des phénomènes de groupement scientifique. Que signifie cette diversité sémantique ? Ces nouveaux éléments nous encouragent à interroger l'émergence des groupes (communautés ou collectifs) qui supportent des communs scientifiques. En 
suivant Weick (1995), on énoncera que le temps de l'émergence d'un groupe est comparable à celui de son fonctionnement ultérieur dans la mesure où les cadres organisationnels existent en grande partie au travers des histoires qui s'y rapportent. Le sens que chacun définit pour ses actions se construit dans la narration. Mais l'identité commune ne constitue qu'une possibilité d'existence des groupes parmi de multiples autres. Dans ces conditions, quels sont les moyens pour faire converger de multiples processus d'identification et de narrations vers un fonctionnement commun?

\section{Production lexicale et régulation des groupes}

En complément des théories de la socialisation, nous avançons que l'un des analyseurs pertinents réside dans le langage et en particulier dans les énoncés qu'affiche un groupe afin de faciliter l'adhésion de ses membres. Nous faisons référence au «tournant linguistique » en sciences sociales (Phillips, Oswick, 2012). Il consiste à analyser les formes discursives comme processus d'élaboration de l'identité des organisations (Czarniawska, 1997 ; Fiol, 2002). Les méthodes d'étude sont encore fort diverses et ne permettent pas de présenter un tableau d'analyse stabilisé (Barberio, Monti, 2014). Les communautés, et a fortiori les communs, n'ont pas encore fait l'objet de ce type d'investigation. Au sein des énoncés, nous focalisons l'étude sur les imaginaires linguistiques. Lexplicitation du pouvoir heuristique de cette option d'analyse passe par des emprunts à plusieurs courants des sciences sociales : les théories de l'activité et celles de la communication.

\section{Théories de l’activité et langage}

Les théories de l'activité se proposent d'initier la compréhension des organisations à partir des unités de base que sont les situations vécues par les personnes au cours de leur activité. On ne s'intéresse pas à l'action programmée ou attendue mais à l'« action située » selon les termes de l'anthropologue Suchman (1987) qui introduit cette expression afin de rendre compte de la conduite d'une action. Cette approche traite d'action située, de cours d'action et de clinique de l'activité.

Un « cours d'action » constitue la représentation du processus complexe que suivent les personnes dans des circonstances matérielles et sociales précises afin de réaliser un ensemble d'actions pertinentes au sein de la situation 
dans laquelle elles sont placées. Ces actions ne suivent pas un plan rationnel, elles mettent plutôt à profit différentes circonstances matérielles et sociales.

Dans ces circonstances, le langage constitue une ressource centrale pour contextualiser les situations car des significations partagées sont requises pour soutenir les communications et engager l'action. Au sein de ces théories de l'activité, certains auteurs relativisent le rôle joué par la situation dans la conduite de l'action afin de ne pas la transformer en facteur déterministe (Clot, 2004). Ils se montrent aussi plus ouverts au sujet des situations de communication. L'incompréhension n'est pas considérée comme une limite à la communication, elle peut constituer une source motrice du développement de la communication (Bakhtine, 1993). Suivant ces auteurs, la compréhension se nourrit de l'incompréhension.

Les actions supposent de la conscience partagée, non pas dans un sens consensuel ou unanimiste, mais dans une perspective ouverte aux divergences d'interprétation et aux incompréhensions. Si la communauté de signification est toujours poursuivie par les membres les plus influents d'un groupe, les différences de sens sont souvent présentes et, de surcroît, elles peuvent jouer un rôle moteur pour poursuivre la communication (Clot, 2004).

\section{Théories de la communication et virtualité des organisations}

Nous nous référons aux théories qui s'ajustent assez finement aux théories de l'activité en poursuivant l'interrogation au sujet des possibilités de communication (Livet, 1994). Le constat de départ est que l'information sur l'ensemble d'un groupe est toujours très incomplète. L'une des rares certitudes est qu'il existe des interactions entre quelques individus. "Dans ses efforts pour communiquer et coordonner ses actions avec celle des autres, l'individu rencontre une limite insurpassable: les intentions d'autrui ne peuvent être absolument garanties. Les raisonnements et les inférences qui devraient déterminer quelles sont les véritables intentions d'autrui concluent à leur propre indécidabilité. Mais cette impuissance des individus leur permet justement de constituer des collectifs » (Livet, 1994, p. 8). Chacun est conduit à supposer une fiction dont il se dit que tous les autres la supposent également. Dans ce sens, un collectif reste virtuel au sens où il représente une supposition d'action et d'identité. L'individu doit donc parier sur ce que sont les repères auxquels les autres se fient.

Ces repères qui rencontreraient l'adhésion d'autrui sont des conventions au sens de Hume (nous n'irons pas jusqu'au courant de l'économie des conventions). Une convention est un repère, implicite ou explicite, dont nous 
supposons tous que les autres aussi l'ont choisi sans que rien dans nos interactions ne démente cette référence. Une convention n'est ni choisie au hasard, ni arbitraire car chacun doit pouvoir s'attendre à ce que les autres reconnaissent lesdits repères.

Pour les personnes en position de direction ou pour celles qui souhaitent influencer le plus grand nombre, la question revient alors à décider de l'animation de cette fiction de groupe. C'est dans le rapport à la langue que se trouvent ces fictions (Hjelmslev, 1942).

\section{La production lexicale participe à la structuration des communautés langagières}

Girin (1990) rappelle que le langage est un outil d'élaboration des représentations qui fonctionne de manière collective au sein des organisations. Les fonctions cognitives et de communication sont intrinsèquement liées. La fonction de communication du langage est biface : elle est orientée vers une activité particulière et vers l'ordre de la société. Les actes de communication produisent des représentations sur les objets et sur les relations (fonction de symbolisation). Symboliser nécessite plusieurs opérations cognitives. Girin identifie quatre types d'opérations d'énonciation (apprendre, produire un lexique, élaborer un propos, interpréter de manière collective). Ces énonciations font exister les groupes humains et structurent en particulier les communautés langagières.

Le concept de grammaire langagière rend compte de la dynamique des communautés langagières (Ferry, 1991, 2004). Il s'agit de rendre compte des processus d'élaboration des discours par les personnes au sujet de leur expérience. Les vécus font l'objet de récits de mieux en mieux élaborés qui forment au fil du temps des discours qui construisent à leur tour l'expérience. Un lien cumulatif s'instaure entre cognition et langage. Les connaissances produites dans et par les grammaires langagières fournissent une contribution à la mise sur pied de communautés d'acteurs. Elles font l'objet de controverses collectives et de révisions. Une analyse sociologique devient alors possible.

Pour sa part, l'activité de symbolisation se définit comme une activité publique, collectivement constituée et reposant sur des conventions (Hajek, 2011). Deux interlocuteurs ne sont pas seuls dans un face-à-face. Ils sont immergés dans un contexte qui nécessite et autorise différentes compétences. Produire du sens prend la forme d'une composante pragmatique (et non pas seulement perceptive). C'est-à-dire que dans un contexte particulier, une compétence d'énonciation et de symbolisation est toujours requise. Les 
grammaires ainsi décrites permettent d'accéder à la dynamique des groupes qui nous intéressent.

\section{Le rôle des imaginaires linguistiques}

Les communautés langagières sont accessibles à l'observateur immergé au sein du groupe. Les communs scientifiques ne se prêtent pas aussi facilement à ce type d'immersion et de recherche en présence des acteurs. Aussi, nous développons une voie exclusivement centrée sur les énoncés produits par ces groupes. L'analyse des imaginaires linguistiques est bien ajustée à ce type d'investigation. Dans la même perspective que celle des communautés langagières, le modèle de l'Imaginaire linguistique (IL) se propose de rendre compte du rapport du sujet à sa langue (Houdebine, 1985, 1993). Inspiré par le lien imaginaire culturel - imaginaire linguistique (Martinet, 1969), et par le rôle des représentations sociales de la langue dans l'évaluation même de la langue (normes et fictions au sens de Hjelmslev, 19421'), ce modèle articule plusieurs niveaux d'expression d'un groupe humain conduisant une activité au travers de normes de langage.

Houdebine définit deux types de normes linguistiques : les normes objectives issues de la description des productions verbales, et les normes subjectives relevant des opinions, jugements, attitudes ou représentations sociales pour les sociologues (Houdebine, 2013). Ces normes sont très utiles pour caractériser l'usage linguistique des différents groupes (Remysen, 2011).

Les normes objectives se répartissent en normes systémiques et normes statistiques (tableau 1). Les premières représentent la conformité des usages aux règles de la structure de la langue. Les secondes concernent leur fréquence. Ces normes peuvent converger ou diverger, ce qui rend compte de la dynamique synchronique des langues.

Les normes subjectives expriment l'imaginaire linguistique proprement dit des locuteurs : c'est le rapport des sujets à leur langue. Cet imaginaire prend la forme d'attitudes parmi lesquelles on peut distinguer différentes normes : évaluatives, fictives, prescriptives, communicationnelles et identitaires.

1. La norme n'est qu'une abstraction qui permet de poser les cadres à la description d'un usage. 
Tableau 1 - L'argumentation en fonction des normes linguistiques

\begin{tabular}{|c|c|c|}
\hline \multicolumn{2}{|c|}{ Normes } & Styles d'arguments \\
\hline \multirow{2}{*}{ 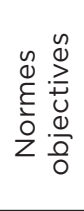 } & $\begin{array}{l}\text { Normes } \\
\text { systémiques }\end{array}$ & $\begin{array}{c}\text { Arguments relatifs à la langue comme système, } \\
\text { autrement dit en tant que code structuré à diffé- } \\
\text { rents niveaux (sémantique, grammatical } \\
\text { et morphologique). }\end{array}$ \\
\hline & $\begin{array}{c}\text { Normes } \\
\text { statistiques }\end{array}$ & Arguments concernant la fréquence d'un emploi. \\
\hline \multirow{5}{*}{ 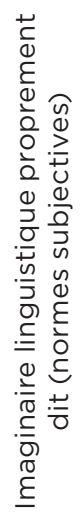 } & $\begin{array}{l}\text { Normes } \\
\text { évaluatives }\end{array}$ & $\begin{array}{l}\text { Correspondent à la conscience que les sujets } \\
\text { possèdent vis-à-vis de la présence ou de l'absence } \\
\text { d'un fait de langue dans leurs usages ou ceux des } \\
\text { autres (cette conscience est fondée ou pas). }\end{array}$ \\
\hline & $\begin{array}{l}\text { Normes } \\
\text { fictives }\end{array}$ & $\begin{array}{l}\text { Organisent les attitudes qui s'appuient sur des } \\
\text { jugements d'ordre esthétique, affectif ou histo- } \\
\text { rique (exemple : l'expression « ça sonne mieux »). }\end{array}$ \\
\hline & $\begin{array}{l}\text { Normes } \\
\text { prescriptives }\end{array}$ & $\begin{array}{c}\text { Sont le résultat d'un processus d'institutionnalisa- } \\
\text { tion (dictionnaires et grammaires), par l'école et les } \\
\text { académies de la langue. }\end{array}$ \\
\hline & $\begin{array}{l}\text { Normes } \\
\text { communica- } \\
\text { tionnelles }\end{array}$ & $\begin{array}{c}\text { Reposent sur la prise en compte du destinataire } \\
\text { dans les échanges (adaptation du langage selon les } \\
\text { contextes). }\end{array}$ \\
\hline & $\begin{array}{l}\text { Normes } \\
\text { identitaires }\end{array}$ & $\begin{array}{c}\text { Assurent la construction de l'identité culturelle } \\
\text { d'une communauté de langue. }\end{array}$ \\
\hline
\end{tabular}

Source: d'après Remysen, (2011, p. 25).

Les deux ensembles de normes (objectives et subjectives) sont en interaction. Elles permettent l'identification, la compréhension et l'élaboration collective des connaissances pour les acteurs d'un groupe. C'est sur cette base de linguistique et d'imaginaire que nous proposons de traiter du mode d'existence et d'exercice des groupes qui soutiennent les communs scientifiques.

\section{Identifier les groupes au moyen de réseaux sémantiques}

La dynamique des groupes est très rarement étudiée en termes linguistiques, encore moins en termes d'imaginaire. Nous utilisons une analyse de discours qualifiée de "socio-sémantique ». On considère deux dimensions dans les énoncés prélevés dans les textes de présentation suffisamment longs des différents groupes sur leur site Internet : le contexte d'énonciation et les caractéristiques lexicales et sémantiques.

Nous disposons d'une base de cas (tableau 2) composée de sociétés savantes, d'associations scientifiques et de groupes de diffusion de l'information scientifique et technique. Cette base de cas a été constituée en 
sélectionnant, d'une part, des associations académiques et scientifiques dont tout nous portait à croire qu'elles répondaient à la caractérisation de " communauté épistémique » ou "de connaissance », d'autre part, des associations en partie constituées de membres scientifiques, ou portant en partie également des problématiques scientifiques. Ces groupes ont été intégrés afin de déceler des comportements ou des déclarations dont nous supposions qu'ils pouvaient être substantiellement différents des premiers. Chacun des groupes est dans une situation d'énonciation identique aux autres. Nous n'avons rien modifié aux textes d'autoprésentation dans chacun des cas.

Nous avons focalisé l'examen sur la dimension des caractéristiques lexicosémantiques. Le canevas de normes linguistiques de Houdebine (tableau 1) nous a permis de différencier les groupes. Le cœur de l'analyse est constitué d'une analyse d'utilisation du lexique, de la synonymie et des champs sémantiques qui y sont associés.

Dans un premier temps, nous avons examiné les textes suivant la fréquence d'apparition des lexèmes utilisés dans l'autoprésentation avec le logiciel Antconc ${ }^{\mathcal{O}}$. Ce logiciel permet de conduire des analyses textuelles. Proxémie $\left(\right.$ CNRTL-Ortolang ${ }^{\odot}$ ) est la seconde ressource d'analyse linguistique utilisée. Elle permet d'explorer les réseaux sémantiques de synonymie. Construit à partir de la compilation de sept dictionnaires de synonymes, Proxémie ${ }^{\odot}$ permet d'analyser les différentes associations sémantiques selon leur «force » ou leur « poids » dans le «système langue », et plus spécifiquement dans les réseaux de synonymie. Nous avons ainsi analysé les liens de proximité sémantique dans le lexique fourni par Proxémie (Cnrtl-Ortolang $\left.{ }^{\odot}\right)$ pour parcourir les réseaux sémantiques attachés aux termes les plus fréquents utilisés dans les différents textes.

Sur cette base, nous étudions, d'une part, la fréquence d'utilisation des termes clés des entités, d'autre part, les réseaux sémantiques associés à ces termes, afin de montrer de quelle manière les entités en question se définissent aussi bien explicitement qu'implicitement.

Le logiciel Antconc ${ }^{\mathbb{C}}$ permet d'analyser les textes en termes de fréquence d'apparition des formes lexicales ${ }^{2}$. Sur cette base, nous avons développé une analyse du poids des six mots les plus fréquents.

Notre hypothèse pose que les mots les plus fréquents de chacun des textes permettent d'élaborer un profil sémantique pour chacun des groupes supportant les communs. Ce nombre a été choisi à partir du constat qu'il n'était pas possible de considérer les mots en deçà de ce seuil comme suffisamment fréquents et représentatifs du propos central du texte. À partir de ce profil

2. Sont exclus les noms propres et les mots en langue étrangère. 
sémantique, nous pouvons établir les objectifs et enjeux qui sous-tendent l'action des différents groupes.

Sur la base des mots les plus utilisés, nous avons identifié les réseaux sémantiques attachés à chaque terme, cela en respectant le champ sémantique du terme cohérent avec le texte ${ }^{3}$. En particulier, nous avons retenu les réseaux de synonymes établis à l'aide de Proxémie (Cnrtl-Ortolang $\left.{ }^{\circledR}\right)$. Les associations générées recouvrent selon nous celles globalement établies par les locuteurs, car la ressource Proxémie ${ }^{\circledR}$ s'appuie sur des dictionnaires de synonymes, eux-mêmes construits par des lexicographes à partir de corpus oraux et écrits.

Une fois ces associations établies, nous avons analysé les champs sémantiques des synonymes afin de faire émerger un profil lexico-sémantique pour chaque groupe. Ce profil est projeté dans la grille des imaginaires linguistiques de Houdebine. Nous avons réalisé ce protocole en aveugle sans identification a priori des groupes. Cette analyse présente uniquement des réseaux sémantiques de synonymes faisant émerger des profils lexicaux et sémantiques des textes de présentation.

En résumé, nous procédons en deux temps pour caractériser les groupes. Dans un premier temps, nous comptabilisons les six mots les plus fréquents pour chacun des groupes (tableau 3) et nous établissons à l'aide des logiciels mentionnés leurs réseaux de synonymes. Dans un second temps, nous analysons les textes d'autoprésentation des groupes à l'aide de ces réseaux de synonymes. Cette analyse nous permet d'apprécier la qualité de chacune des sept normes de Houdebine. Cette appréciation n'est pas automatique, ni algorithmique. Elle procède de notre lecture des textes assistée des premiers traitements quantitatifs (tableau 4).

Parmi les vingt-trois cas analysés, six ont été choisis au hasard afin d'illustrer les formes qu'y prennent les normes définies par Houdebine. Les informations relatives à chaque niveau de norme sont par la suite examinées et regroupées en types linguistiques, ce qui nous permet d'identifier des ensembles d'énoncés homogènes (tableau 5).

Tableau 2 - Les groupes étudiés

\begin{tabular}{|c|c|}
\hline Appellations officielles & Adresse \\
\hline 350.org & http://350.org/fr/ \\
\hline $\begin{array}{c}\text { April, promotion et recherche en } \\
\text { informatique libre }\end{array}$ & https://www.april.org/ \\
\hline
\end{tabular}

3. En cas de polysémie d'un terme, l'exploration est ramenée au champ sémantique approprié au contexte : « cadre » sera analysé dans le champ de «milieu » et « entourage », et non dans le champ « châssis ». 


\begin{tabular}{|c|c|}
\hline Appellations officielles & Adresse \\
\hline $\begin{array}{c}\text { Association Française de } \\
\text { Marketing }\end{array}$ & http://www.afm-marketing.org/ \\
\hline $\begin{array}{c}\text { Asso Internationale pour } \\
\text { une Médecine Scientifique } \\
\text { Indépendante et Bienveillante }\end{array}$ & https://aimsib.org/presentation-de-laimsib/ \\
\hline Agecso & http://www.agecso.com/wp/ \\
\hline Alterondes & http://www.alterondes35.org/ \\
\hline AnimFr & http://imaginationforpeople.org/wiki/work- \\
\hline group/animfr/animfrinfo
\end{tabular}

\section{Imaginaires des communs scientifiques: deux orientations différentes}

Notre analyse s'inscrit à mi-chemin entre « légitimité externe » et " légitimité interne » des organisations. La légitimité externe a été beaucoup plus étudiée que la légitimité interne (Clegg et al., 2007). Dans notre recherche les deux dimensions interne et externe doivent être abordées en continuité : d'une part, en raison du média numérique qui instaure cette continuité, d'autre part, parce qu'il est vital pour ces groupes de diffuser un discours « externe » pour recruter de nouveaux membres «en interne ». Ces deux 
dimensions sont tout à fait compatibles avec notre question de départ au sujet de la coordination au sein des groupes de pairs : de quelle manière les conventions linguistiques facilitent l'intercompréhension et la cohésion des actions au sein des groupes?

\section{Référence à l'autorité procédurale ou dynamique sociétale}

Lanalyse des six mots les plus utilisés et de leurs synonymes fait apparaître des formes d'expression assez différentes entre les différents groupes. Le tableau 3 présente dix illustrations parmi les vingt-trois cas étudiés. Certains groupes font appel à des termes à visée objective (article, conférence, recherche, revue, scientifique, université) faisant directement référence à une activité académique et à son autorité procédurale alors que d'autres font référence à l'action au sein de l'espace public (contribuer, acteurs, habitants, institutions, conseil, impact, travail), parfois même directement à la notion de communs. La question du pouvoir et des enjeux est également présente dans cette forme d'autoprésentation ainsi que les thèmes " conflit », " pouvoir » et « indépendance ».

Cependant l'examen détaillé des vingt-trois textes de présentation des groupes fait apparaître une certaine confusion dans l'usage des termes communauté et collectif. Le premier terme est régulièrement utilisé comme synonyme du second. L'autoprésentation des différents groupes entretient la confusion dans l'emploi de ces deux termes. Nous retrouvons le trouble rencontré au sein des travaux en sociologie des sciences qui définissent du collectif par du communautaire, ou bien du communautaire par du fonctionnement collectif. Tous les groupes étudiés ne nous aident pas à ce stade pour dissiper ce trouble.

Le premier résultat qui apparaît clairement concerne une distinction entre un ensemble de groupes académiques (que l'on peut qualifier sans usurpation de " communautés académiques ») et un ensemble de groupes dont le point commun est justement de ne pas s'auto-présenter comme groupes exerçant une activité académique.

Dans cet ensemble, certains évoquent un champ particulier au sein duquel quelques-uns de leurs membres exercent (médecine, santé, logiciel, numérique, urbanisme, ville) sans toutefois s'y limiter. Souvent ces qualificatifs sont complétés de verbes d'action ou d'une référence à l'espace public. 
Tableau 3 - Mots les plus fréquents dans les autoprésentations de dix groupes parmi les vingt-trois étudiés * (pour chaque mot les synonymes les plus fréquents apparaissent par ordre)

\begin{tabular}{|c|c|c|c|c|c|}
\hline \multicolumn{6}{|c|}{ Texte AFFI (Word Types: 493; Word Tokens: 1267) } \\
\hline finance & $\begin{array}{c}\text { associa- } \\
\text { tion }\end{array}$ & revue & conférence & membre & article \\
\hline argent & réunion & publication & colloque & adhérent & sujet \\
\hline bourse & société & périodique & table ronde & cotisant & objet \\
\hline banque & Union & publi & congrès & participant & chapitre \\
\hline affaires & $\begin{array}{l}\text { groupe- } \\
\text { ment }\end{array}$ & & symposium & souscripteur & thème \\
\hline économie & alliance & & assises & & point \\
\hline \multicolumn{6}{|c|}{ Texte Économistes attérés (Word Types: 438; Word Tokens: 1514) } \\
\hline association & $\begin{array}{l}\begin{array}{c}\text { écono- } \\
\text { mique }\end{array} \\
\end{array}$ & $\begin{array}{l}\text { écono- } \\
\text { mistes }\end{array}$ & atterrés & article & conseil \\
\hline réunion & $\begin{array}{l}\text { gestion- } \\
\text { naire }\end{array}$ & & consterné & sujet & $\begin{array}{l}\text { assem- } \\
\text { blée }\end{array}$ \\
\hline société & $\begin{array}{l}\text { managé- } \\
\text { rial }\end{array}$ & & abattu & objet & aréopage \\
\hline union & & & accablé & chapitre & chambre \\
\hline groupement & & & catastrophé & thème & bureau \\
\hline alliance & & & anéanti & point & voix \\
\hline \multicolumn{6}{|c|}{ Texte Cortecs (Word Types: 312; Word Tokens: 665) } \\
\hline $\begin{array}{c}\text { esprit cri- } \\
\text { tique }\end{array}$ & contribuer & $\begin{array}{c}\begin{array}{c}\text { pouvoir } \\
\text { (VB) }\end{array} \\
\end{array}$ & conflit & d'intérêt & formation \\
\hline \multirow[t]{5}{*}{ sens critique } & aider & $\begin{array}{c}\text { être en } \\
\text { mesure de }\end{array}$ & divergence & avantage & $\begin{array}{l}\text { instruc- } \\
\text { tion }\end{array}$ \\
\hline & participer & $\begin{array}{l}\text { avoir le } \\
\text { droit de }\end{array}$ & litige & bien & culture \\
\hline & coopérer & $\begin{array}{l}\text { avoir la } \\
\text { possibilité } \\
\text { de }\end{array}$ & différend & dividende & savoir \\
\hline & collaborer & $\begin{array}{c}\text { être } \\
\text { capable de }\end{array}$ & contentieux & bénéfice & bagage \\
\hline & concourir & & désaccord & revenu & éducation \\
\hline \multicolumn{6}{|c|}{ Texte AFM (Word Types: 379; Word Tokens: 966) } \\
\hline marketing & $\begin{array}{c}\text { internatio- } \\
\text { nal }\end{array}$ & $\begin{array}{c}\begin{array}{c}\text { scienti- } \\
\text { fique }\end{array} \\
\end{array}$ & recherche & université & $\begin{array}{l}\text { associa- } \\
\text { tion }\end{array}$ \\
\hline mercatique & universel & chercheur & $\begin{array}{l}\text { reconnais- } \\
\text { sance }\end{array}$ & académie & $\begin{array}{l}\text { confédé- } \\
\text { ration }\end{array}$ \\
\hline marchéage & $\begin{array}{c}\text { multilaté- } \\
\text { ral }\end{array}$ & rationnel & examen & école & $\begin{array}{l}\text { partena- } \\
\text { riat }\end{array}$ \\
\hline $\begin{array}{c}\text { commerciali- } \\
\text { sation }\end{array}$ & mondial & savant & exploration & $\begin{array}{c}\text { établisse- } \\
\text { ment }\end{array}$ & tontine \\
\hline \multirow[t]{2}{*}{$\begin{array}{l}\text { merchandi- } \\
\text { sing } \\
\end{array}$} & plurilatéral & théorique & étude & fac & colonie \\
\hline & & $\begin{array}{l}\text { métho- } \\
\text { dique }\end{array}$ & prospection & faculté & affiliation \\
\hline
\end{tabular}




\begin{tabular}{|c|c|c|c|c|c|}
\hline \multicolumn{6}{|c|}{ Texte Savoirs Com1 (Word Types: 360; Word Tokens: 784) } \\
\hline manifeste & collectif & numérique & règles & biens & communs \\
\hline \multirow[t]{2}{*}{$\begin{array}{c}\text { profession } \\
\text { de foi }\end{array}$} & équipe & digital & loi & possession & partagé \\
\hline & ensemble & numéral & $\begin{array}{l}\text { principes } \\
\text { règlement }\end{array}$ & & collectif \\
\hline \multicolumn{6}{|c|}{ Texte Asso Inter Médecines (Word Types: 244; Word Tokens: 497) } \\
\hline médecine & santé & $\begin{array}{l}\text { scienti- } \\
\text { fique }\end{array}$ & $\begin{array}{c}\text { indépen- } \\
\text { dante }\end{array}$ & $\begin{array}{c}\text { profession- } \\
\text { nels }\end{array}$ & adhérents \\
\hline remède & équilibre & chercheur & autonome & technicien & membre \\
\hline médicament & hygiène & rationnel & souverain & spécialiste & $\begin{array}{c}\text { partici- } \\
\text { pant }\end{array}$ \\
\hline traitement & $\begin{array}{c}\text { constitu- } \\
\text { tion }\end{array}$ & savant & libre & & adepte \\
\hline $\begin{array}{l}\text { thérapeu- } \\
\text { tique }\end{array}$ & force & théorique & libéré & & associé \\
\hline chirurgie & $\begin{array}{c}\text { tempéra- } \\
\text { ment }\end{array}$ & $\begin{array}{l}\text { métho- } \\
\text { dique }\end{array}$ & affranchi & & inscrit \\
\hline \multicolumn{6}{|c|}{ Texte Collectif surexposition écrans (Word Types: 363; Word Tokens: 850) } \\
\hline écrans & enfants & collectif & difficultés & $\begin{array}{c}\text { profession- } \\
\text { nels }\end{array}$ & santé \\
\hline \multirow[t]{5}{*}{ moniteur } & gamin & équipe & obstacle & technicien & équilibre \\
\hline & môme & ensemble & embarras & spécialiste & hygiène \\
\hline & rejeton & & $\begin{array}{c}\text { empêche- } \\
\text { ment }\end{array}$ & & $\begin{array}{l}\text { constitu- } \\
\text { tion }\end{array}$ \\
\hline & $\begin{array}{l}\text { progéni- } \\
\text { ture }\end{array}$ & & anicroche & & force \\
\hline & $\begin{array}{l}\text { descen- } \\
\text { dant }\end{array}$ & & contrariété & & \\
\hline \multicolumn{6}{|c|}{ Texte Collectif Bazar urbain (Word Types: 655; Word Tokens: 2148) } \\
\hline projet & acteurs & lieu & $\begin{array}{c}\text { représenta- } \\
\text { tions }\end{array}$ & travail & habitants \\
\hline dessein & $\begin{array}{l}\text { protago- } \\
\text { niste }\end{array}$ & endroit & image & tâche & résident \\
\hline plan & & place & symbole & affaire & citoyen \\
\hline intention & & $\begin{array}{c}\text { emplace- } \\
\text { ment }\end{array}$ & dessin & occupation & $\begin{array}{l}\text { popula- } \\
\text { tion }\end{array}$ \\
\hline esquisse & & coin & schéma & fonction & personne \\
\hline ébauche & & situation & plan & activité & individu \\
\hline \multicolumn{6}{|c|}{ Texte Collectif labos1 point5 (Word Types: 492; Word Tokens: 1276) } \\
\hline collectif & recherche & travail & impact & scientifique & empreinte \\
\hline équipe & examen & tâche & effet & chercheur & marque \\
\hline \multirow[t]{4}{*}{ ensemble } & $\begin{array}{l}\text { explora- } \\
\text { tion }\end{array}$ & affaire & influence & rationnel & trace \\
\hline & enquête & occupation & conséquence & savant & signe \\
\hline & étude & fonction & retombées & théorique & stigmate \\
\hline & $\begin{array}{l}\text { investiga- } \\
\text { tion }\end{array}$ & activité & & méthodique & influence \\
\hline
\end{tabular}




\begin{tabular}{|c|c|c|c|c|c|}
\hline \multicolumn{7}{|c|}{ Texte April (Word Types: 240; Word Tokens: 621) } \\
\hline logiciel & libre & action & association & enjeux & $\begin{array}{c}\text { institu- } \\
\text { tions }\end{array}$ \\
\hline programme & gratuit & travail & réunion & objectif & $\begin{array}{c}\text { gouver- } \\
\text { nement }\end{array}$ \\
\hline software & disponible & affaire & société & & $\begin{array}{c}\text { adminis- } \\
\text { tration }\end{array}$ \\
\hline application & $\begin{array}{c}\text { indépen- } \\
\text { dant }\end{array}$ & tâche & union & & autorités \\
\hline & autonome & procès & groupement & & \\
\hline & & activité & alliance & & \\
\hline
\end{tabular}

* Les noms propres, les mots adoptés d'autres langues, et les mots n'ayant pas d'entrée dans la ressource Proxémie (Cnrtl-Ortolang $\left.{ }^{\circledR}\right)$ n'ont pas été retenus.

\section{Diversité des normes linguistiques et orientation de l’intérêt}

Lanalyse des imaginaires linguistiques permet d'explorer plus précisément les conventions linguistiques en usage au sein de chacun des groupes. Sur cette base, elle pourrait participer à l'élaboration d'une nouvelle typologie en complément de celles qui différencient communautés de pratique et communautés épistémiques (Cohendet, Llerena, 2003).

Les imaginaires linguistiques s'articulent en normes objectives et normes subjectives, elles sont présentées pour six groupes aux profils contrastés (tableau 4).

Les normes objectives confèrent une consistance officielle au groupe qui se présente au public. La norme systémique sera abondamment utilisée par une société savante qui souhaitera afficher des termes qui renvoient aux systèmes institutionnels (établissements, entreprises, organisations, disciplines). Alors que d'autres groupes qui affirment très explicitement leur orientation vers le public n'useront pas du même vocabulaire. Leurs normes systémiques et statistiques sont faiblement informées par leurs textes d'autoprésentation recueillis sur leurs pages Internet. La référence à la validation sociale du savoir par l'institution académique y est faiblement présente. Il est plutôt question d'utilité sociale.

Les normes statistiques font apparaître que les associations et sociétés savantes attachent de l'importance à leur nombre d'adhérents, à leur couverture internationale ainsi qu'au volume de production scientifique. Les groupes davantage engagés dans un imaginaire politique présentent un bien moindre intérêt pour ce type de dénombrement. 
Toutefois il sera difficile d'établir une segmentation de notre base de cas sur la base de ces normes statistiques. Plusieurs associations et sociétés savantes que l'on pourrait qualifier de communautés académiques rassemblent un grand nombre de membres, alors que d'autres groupes que l'on pourrait qualifier de collectifs engagés dans des causes environnementales, sociales et politiques ne sont pas forcément de grands réseaux d'individus, mais souvent des groupes de taille réduite.

En termes plus qualitatifs, les normes subjectives éclairent davantage les différenciations entre les groupes. C'est à ce niveau que les conventions linguistiques semblent prendre leur pleine expression au travers des cinq autres normes. Les tableaux 4 et 5 présentent notre interprétation des expressions les plus pertinentes pour chaque groupe aux différents niveaux de ces normes subjectives.

Les groupes qui se rapprochent le plus de l'univers académique mettent en avant le progrès scientifique et la qualité de l'enseignement, l'excellence des travaux qu'ils conduisent et la dimension internationale. Ces groupes académiques emploient des termes qui tendent à les présenter comme porte-parole d'une légitimité qui ne se prête pas à discussion. Leur intérêt consiste à valoriser et diffuser. Les autres groupes marquent une distance vis-à-vis de cette orientation. Ils semblent majoritairement se référer à "l'esprit critique », la " diversité » des acteurs et des médias, ainsi qu’à la dimension «projet » de leurs activités. Dans certains cas, un intérêt est marqué pour « l'expression publique » et les « communs de la connaissance ».

À partir d'un examen particulier des vingt-trois cas sur la base des normes subjectives, nous avons établi une dichotomie de la totalité de notre population (tableau 5). Le premier ensemble est dénommé « communautés » dans le sens où ce qui est affiché désigne majoritairement un rassemblement de pairs animés par les valeurs d'« excellence » et de progrès scientifique, et visant la notoriété internationale. Les cas qui entrent dans cette dénomination sont : AFFI, AFM, AIM, Société Française de Physique, Association Informatique Médicale, Société Informatique de France.

Le second ensemble appelé « collectifs » fait appel à d'autres conventions linguistiques telles que l'« horizontalité » et l'esprit critique, la promotion de valeurs très proches des biens communs, l'indépendance vis-à-vis d'intérêts supposés hostiles à ces biens communs. Dans cet ensemble, la conduite de l'action paraît associée à une pluralité d'acteurs et la notion de réseau, comme espace de l'autorité, est utilisée le plus souvent. Les groupes se rangeant dans cette catégorie de " collectifs » sont : Alterondes, AnimFr, April, Ars Industrialis, Association internationale pour une médecine 


\begin{tabular}{|c|c|c|c|c|c|c|c|c|}
\hline 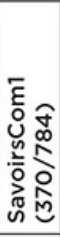 & 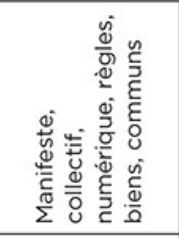 & 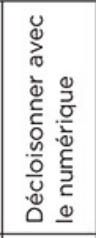 & 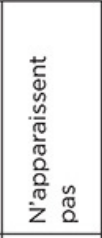 & 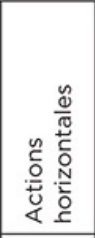 & 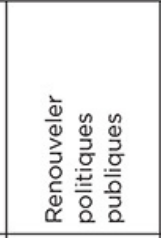 & 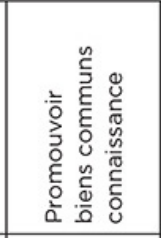 & 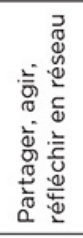 & 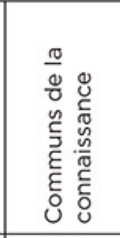 \\
\hline 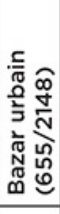 & 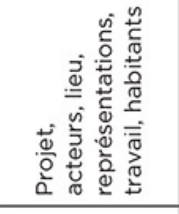 & 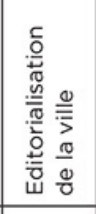 & 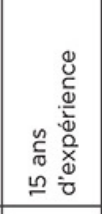 & 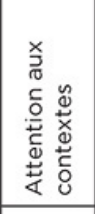 & 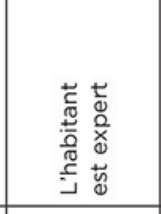 & 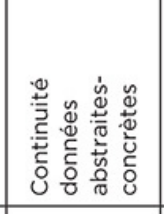 & 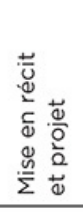 & 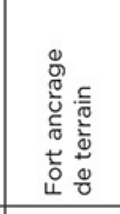 \\
\hline 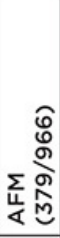 & 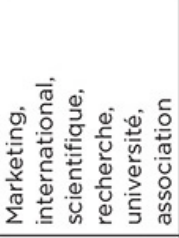 & 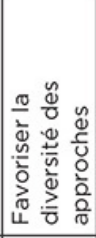 & 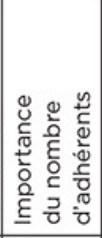 & 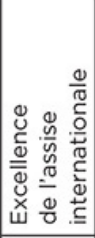 & 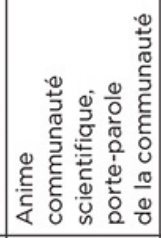 & 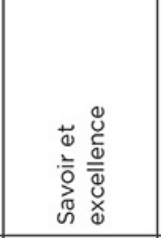 & 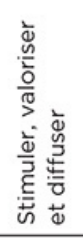 & 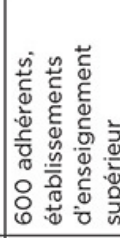 \\
\hline 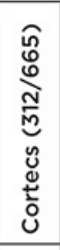 & 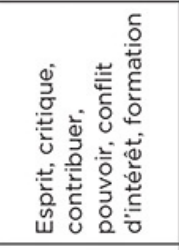 & 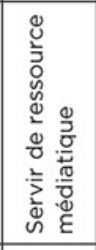 & 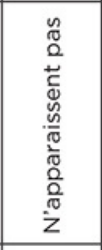 & 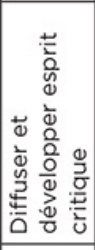 & 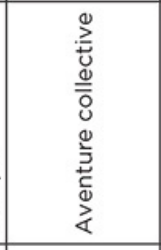 & 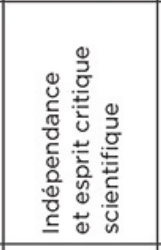 & 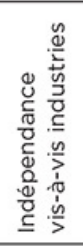 & 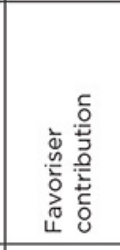 \\
\hline 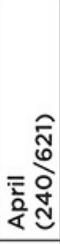 & 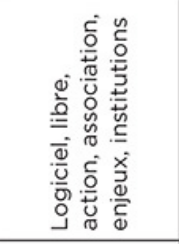 & 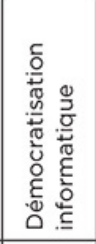 & 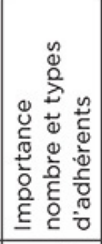 & 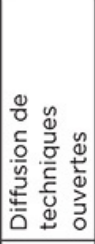 & 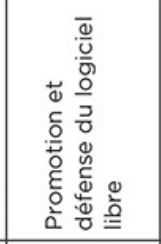 & 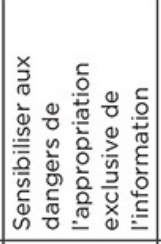 & 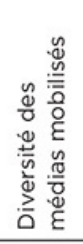 & 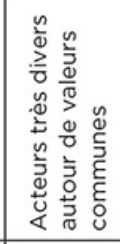 \\
\hline 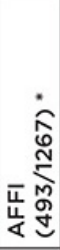 & 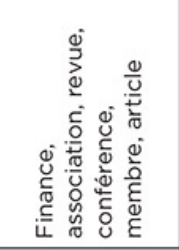 & 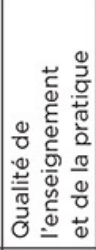 & 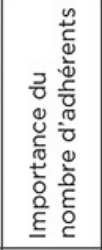 & 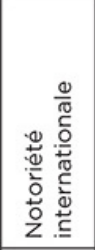 & 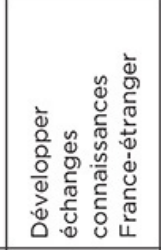 & 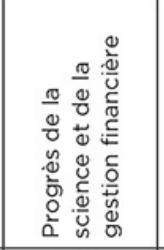 & 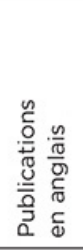 & 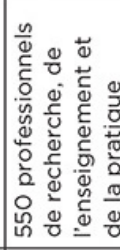 \\
\hline & 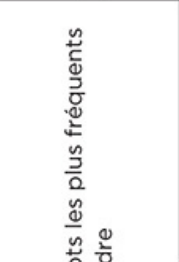 & 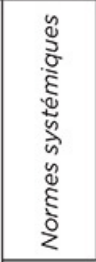 & 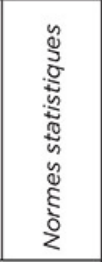 & 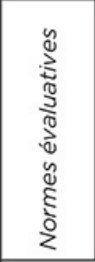 & 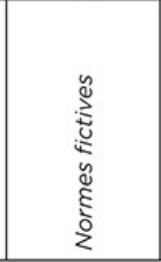 & 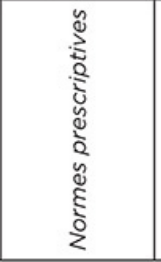 & 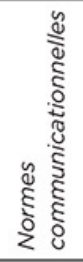 & 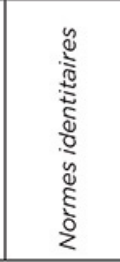 \\
\hline & 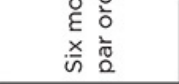 & \multicolumn{2}{|c|}{ 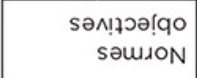 } & \multicolumn{5}{|c|}{ 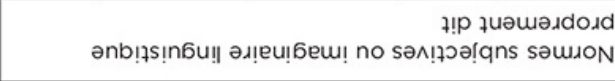 } \\
\hline
\end{tabular}

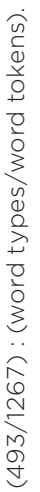


scientifique indépendante, Collectif surexposition écrans, Bazar urbain, Cortecs, Ecoatterrés, Negawatt, SavoirsCom1 et Sharelex.

Un ensemble intermédiaire en termes de conventions linguistiques (Agecso, Knowledge foundation, Collectif labos1 point5) ne répond pas complètement aux propriétés ni de ces communautés, ni des collectifs.

Tableau 5 - Regroupement des cas en deux grands groupes

\begin{tabular}{|c|c|c|c|}
\hline \multicolumn{2}{|r|}{ Normes } & Communautés & Collectifs \\
\hline \multirow[b]{2}{*}{ 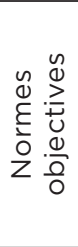 } & $\begin{array}{c}\text { Normes } \\
\text { systémiques }\end{array}$ & $\begin{array}{l}\text { Diversité et qualité de la } \\
\text { discipline }\end{array}$ & $\begin{array}{l}\text { Démocratiser } \\
\text { et décloisonner }\end{array}$ \\
\hline & $\begin{array}{l}\text { Normes } \\
\text { statistiques }\end{array}$ & $\begin{array}{l}\text { Importance du nombre } \\
\text { d'adhérents }\end{array}$ & $\begin{array}{l}\text { N'apparaissent pas } \\
\text { souvent ou bien années } \\
\text { d'expérience ou encore } \\
\text { mention diversité des } \\
\text { adhérents }\end{array}$ \\
\hline \multirow{5}{*}{ 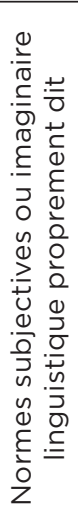 } & $\begin{array}{l}\text { Normes } \\
\text { évaluatives }\end{array}$ & Notoriété internationale & $\begin{array}{c}\text { Diffuser, techniques } \\
\text { ouvertes, horizontalité et } \\
\text { esprit critique }\end{array}$ \\
\hline & $\begin{array}{l}\text { Normes } \\
\text { fictives }\end{array}$ & $\begin{array}{c}\text { Développement interna- } \\
\text { tional de la communauté } \\
\text { scientifique }\end{array}$ & $\begin{array}{c}\text { Renouveler, promouvoir } \\
\text { valeurs et outils dédiés } \\
\text { pour la collectivité }\end{array}$ \\
\hline & $\begin{array}{l}\text { Normes pres- } \\
\text { criptives }\end{array}$ & Excellence et Progrès & $\begin{array}{c}\text { Indépendance vis-à-vis } \\
\text { appropriations exclusives } \\
\text { et promotion des biens } \\
\text { communs }\end{array}$ \\
\hline & $\begin{array}{l}\text { Normes } \\
\text { communica- } \\
\text { tionnelles }\end{array}$ & $\begin{array}{c}\text { Valoriser et diffuser } \\
\text { suivant standards interna- } \\
\text { tionaux }\end{array}$ & $\begin{array}{l}\text { Agir en réseau avec } \\
\text { diversité de médias }\end{array}$ \\
\hline & $\begin{array}{c}\text { Normes } \\
\text { identitaires }\end{array}$ & Nombre de pairs & $\begin{array}{l}\text { Diversité acteurs autour } \\
\text { de valeurs communes }\end{array}$ \\
\hline
\end{tabular}

\section{Ce que peuvent nous apprendre les analyses lexicales au sujet de l'organisation des communs scientifiques}

Les enseignements de notre analyse peuvent s'énoncer selon trois perspectives complémentaires. Tout d'abord, la méthode utilisée pour mettre en évidence des conventions linguistiques au sein des organisations, et en particulier au sein d'activités scientifiques, a fait l'objet de trop rares études. Envisagées d'un point de vue conceptuel (Livet, 1994), les conventions linguistiques au sens de ressources pour rassembler les actions individuelles, n'ont pas encore donné lieu à des constats et analyses empiriques. L'exploration des imaginaires linguistiques (Houdebine) nous semble appropriée pour développer ces recherches et offre des perspectives d'approfondissement fécondes. Lenseignement analytique qui en découle vise les typologies de l'action 
collective. Enfin, un troisième apport concerne justement cette nouvelle catégorie de « collectif » qui se présente comme capacité potentielle de développement de communs scientifiques.

Les enseignements de notre étude prennent leur source dans la méthodologie que nous utilisons. Nous faisons donc de notre heuristique le premier des apports à la connaissance des communs scientifiques. Cette méthode permet de montrer des réseaux sémantiques qui rassemblent soit des synonymes, soit d'autres types d'associations sémantiques (par exemple le mot " article » peut renvoyer à l'un de ses synonymes « chapitre » aussi bien qu'à « lire »).

Les réseaux synonymiques révélés dans Proxémie sont basés sur l'analyse de corpus et de dictionnaires de synonymes, eux-mêmes élaborés à partir de corpus oraux et écrits de la langue française. En d'autres termes, les relations sémantiques apparaissant dans Proxémie se fondent sur l'utilisation de synonymes par divers locuteurs dans différents contextes.

Chez les locuteurs, qu'ils soient en situation de production verbale ou de compréhension, des aires sémantiques doivent par conséquent être partagées, sans quoi l'intercompréhension ne serait pas possible. Cela implique que, lorsqu'un mot $\mathrm{X}$ situé en contexte est activé dans le lexique mental des locuteurs, le réseau synonymique associé à ce mot et activé via le mot $\mathrm{X}$, est partagé par les locuteurs.

Nous considérons que les synonymes rattachés aux lexèmes des plus fréquents des textes de présentation sont représentatifs des aires lexicales activées lors de la lecture de ces textes. À partir des aires lexico-sémantiques ainsi dégagées, il devient dès lors possible d'établir un lien avec l'imaginaire linguistique.

Ce premier apport est de nature méthodologique : l'analyse des caractéristiques lexico-sémantiques de l'imaginaire en acte des communautés est un puissant vecteur de qualification de leur activité.

Notre deuxième apport est de nature typologique. Selon la dichotomie que nous présentons avec le tableau 5, on peut avancer que « faire du commun scientifique » ne signifie pas se comporter de manière similaire suivant les groupes que l'on a rejoint. Rejoindre une association académique consiste effectivement à prendre place au sein d'une communauté scientifique. Pour ce type d'organisation communautaire, il s'agit de s'engager dans une aventure scientifique dont les règles sont connues tant en termes de coordination au sein du groupe de pairs que du point de vue des rétributions du mérite. Lapport de l'activité à la société est implicite. Lactivité académique est consacrée à l'avancée des connaissances au sein d'une discipline particulière dont l'orientation et les règles de certification des connaissances sur longue période sont régulièrement redéfinies (Kuhn, 1962). Il en va différemment 
pour les collectifs qui sont de véritables regroupements hybrides. Un collectif peut rassembler des scientifiques ainsi que d'autres professionnels, ou encore des citoyens, selon des agencements que nous n'analysons pas ici en termes de réseaux de traduction. La sociologie de la traduction (Akrich et al., 2006) n'est pas notre propos. À partir de notre seule analyse textuelle, nous proposons de mettre à distance cette hégémonie de la dénomination « communauté » pour désigner toute forme d'organisation de l'activité qui ne relèverait pas de la figure de la hiérarchie.

Selon nous, il n'existe pas d'homogénéité au sein des groupes qui animent les communs scientifiques. Lanalyse sémantique inspirée par l'imaginaire linguistique fait au contraire apparaître deux grandes catégories d'agir au sein de ces communs.

Nous rejoignons Knorr-Cetina (1999) pour contester cette supposée homogénéité dans la manière de conduire l'activité scientifique. Sans doute le modèle universitaire (la communauté de pairs) a constitué le seul attracteur pour rendre compte de cette activité productive éloignée du monde marchand. Et sans doute également les formes d'hybridation entre différents univers scientifiques et marchands ont-elles été interprétées dans un sens exclusif empêchant des voies complémentaires d'entrer dans le débat (Shinn, 2002). Mais notre propos ne se réduit pas à cette argumentation. Fondé sur un ensemble de cas certes réduit, notre travail soumet une possibilité de différenciation entre des communs articulés à des communautés académiques, et des communs adossés à des collectifs. Nous proposons donc un affinement du vocabulaire employé afin de rendre justice à une modalité de conduite de l'activité scientifique qui ne répond pas aux propriétés affichées des communautés épistémiques. Les collectifs ne sont pas une forme nuancée de communauté épistémique, ils représentent un tout autre projet de communs scientifiques.

Tous les communs que nous avons étudiés n'œuvrent pas dans le même sens, notamment dans leur relation aux usages du savoir et des connaissances. La communauté épistémique ne constitue pas la modalité exclusive de régulation des communs scientifiques. Les collectifs, par exemple, se constituent contre des pratiques qualifiées d'hégémoniques et de néfastes sur un plan sanitaire, social ou politique. La forme communautaire, très souvent concomitante de l'expression académique, consiste à entretenir et préserver une action instituée. Dans ses modalités de coordination, et non dans ses objets d'étude, la communauté apparait ainsi comme une forme conservatrice. Alors que la forme collective, détachée des liens académiques, semble davantage intéressée par le mouvement afin d'instituer de la nouveauté. 
Un troisième apport concerne donc cette forme spécifique de mise en œuvre d'un commun scientifique. Par l'usage de conventions et d'imaginaires linguistiques différents, les collectifs apparaissent comme une alternative forte à la régulation de type académique des communs scientifiques.

Les collectifs se prêtent à une différenciation assez nette vis-à-vis des communautés épistémiques en termes de fonctionnement organisationnel. La lecture du tableau 5 rend sensible cette alternative. Alors que les communautés se donnent un cadre pour la conduite de l'activité, les collectifs font appel au « décloisonnement ", à l'usage de " techniques ouvertes », à un fonctionnement « horizontal » et à des « outils dédiés pour la collectivité ». Il s'agit dans leur cas de rassembler une diversité d'acteurs autour de valeurs communes.

Alors que les objectifs d'une communauté consistent à produire de nouvelles connaissances, les collectifs sont clairement engagés dans une priorité sociétale qui peut avoir besoin de ces connaissances. Les priorités ne sont donc pas les mêmes.

Les activités cognitives d'une communauté épistémique s'organisent autour de la codification des connaissances alors que les collectifs privilégient l'accumulation de preuves afin de conduire une action impliquant des acteurs extrascientifiques. Les communautés recrutent leurs membres au moyen d'une procédure validée par les pairs alors que les collectifs préfèrent souvent l'adhésion aux valeurs. Pour une communauté, il est important que les membres respectent une autorité procédurale alors que les membres d'un collectif sont très attachés à l'adhésion à quelques valeurs proclamées.

Enfin, les incitations s'organisent autour de la reconnaissance par les pairs pour une communauté académique. Au sein d'un collectif, il s'agit de renforcer sans cesse la légitimité des valeurs afin de faciliter la réassurance des adhérents.

Les collectifs se présentent comme des formes sans doute moins stables que les communautés académiques avec lesquelles elles peuvent collaborer notamment au travers de certains acteurs qui partagent leur temps entre ces deux formes d'action collective. Par exemple, des universitaires qui à partir des travaux de leur communauté décident de conduire une action en dehors de la sphère académique en rejoignant les valeurs d'un collectif particulier. Les collectifs ne peuvent pas conduire une activité scientifique de manière indépendante. Ce qui peut être le cas pour certaines communautés académiques qui conduisent leur activité en termes axiomatiques ou spéculatifs. Ces collectifs ont également besoin de publics au nom desquels, et pour lesquels, ils vont tenter de développer des processus de recherche scientifique. 


\section{Conclusion}

Notre intention consistait à mettre en évidence la conduite de l'action collective au sein des communs scientifiques dans une perspective éventuelle de différenciation des types de conduite. Notre démarche consistait à mobiliser un dispositif méthodologique de nature linguistique encore peu utilisé dans notre champ d'étude.

Notre étude peut se résumer par trois principaux apports et une perspective d'approfondissement.

- Renouveler l'analyse des communs scientifiques par l'étude des organisations productives.

- Discuter des usages des énoncés scientifiques pour démontrer la diversité d'exercice de la pratique scientifique.

- Revisiter la problématique des communautés, notamment dans l'univers scientifique, en diversifiant les catégories d'analyse.

Enfin, ces apports sont marqués par des limites en termes d'étendue de l'investigation. Les dépasser permettra d'ouvrir de nouvelles perspectives d'analyse des communs scientifiques et des groupes qui les soutiennent.

Si les travaux se sont focalisés sur les droits d'accès à la ressource en montrant la complexité des faisceaux de droit qui pouvaient être élaborés, il nous semble que les arrangements organisationnels entre commoners ont été négligés. Certes la gouvernance de la ressource est intégralement partie prenante de cette organisation, mais elle ne peut rendre compte à elle seule de l'ensemble des pratiques. Montrer la science en train de se faire consiste à se centrer sur ses processus d'élaboration, et donc sur les moyens techniques utilisés. À partir du moment où ressource et commoners sont consubstantiels, pourquoi ne pas saisir les modes d'organisation des commoners ? Et, partant, s'engager dans une grande typologie des communs scientifiques? Le premier apport de notre étude invite à relever un tel défi. Pour l'heure, l'organisation des pratiques scientifiques fait l'objet d'une typologie formelle, mais pas d'une recension étendue. Nous préconisons pour cela de considérer les techniques mobilisées dans les processus d'élaboration des expériences et des démonstrations scientifiques. Le langage est l'une de ces techniques. Il permet de représenter le monde : ses problèmes, ses manifestations et leurs enjeux. Sans langage il est impossible de développer une activité scientifique.

Le deuxième apport consiste à présenter la pertinence d'une analyse linguistique des organisations, et en particulier des communs. Nous avons fait l'hypothèse que la conduite de l'action collective au sein des communs scientifiques pouvait faire l'objet d'une approche en termes de conventions linguistiques. Les moyens utilisés pour cela sont ceux du langage. Laccent porté sur 
tel ou tel terme oriente l'attention et, par accumulation, fonde des représentations partagées qui vont fonctionner comme de véritables ressources pour la production des résultats scientifiques. La problématique des représentations sociales de la langue fournit un vecteur de différenciation des énoncés. Les imaginaires linguistiques permettent de décliner ces représentations sociales en de multiples niveaux.

L'analyse conduite fait apparaître une certaine diversité de conventions au travers des différents niveaux d'imaginaires linguistiques. Discuter des usages des énoncés scientifiques pour démontrer la diversité d'exercice de la pratique scientifique constitue notre deuxième apport et nous conduit au troisième et dernier enseignement.

Nous développons un examen critique de la catégorie d'analyse qui s'est imposée dans les dernières décennies en économie et gestion de l'innovation. Les communautés sont présentées aujourd'hui comme la modalité alternative exclusive aux organisations hiérarchiques, certes avec une certaine diversité (communautés de pratique, épistémiques, de connaissance et communautés pilotées). Ces distinctions ne suffisent pas à rendre compte des pratiques alternatives aux organisations hiérarchiques. En particulier dans le domaine scientifique, la sociologie des sciences montre que la diversité des pratiques liée aux disciplines, ou parfois simplement aux contextes, incite à la prudence.

Nous pensons pouvoir introduire la pertinence de la catégorie « collectif » dans ses dimensions d'imaginaire linguistique et d'activité cognitive organisée. Dans une inspiration proche de celle de Knorr-Cetina (1999), qui interroge les différentes procédures d'investigation scientifique comme autant de modalités de la science en train de se faire, il nous paraît pertinent d'intégrer l'interrogation politique et sociale au sein même de la problématique de ces groupes qui soutiennent les communs scientifiques. Deux grands ensembles d'imaginaires paraissent se dégager : l'un rejoignant les pratiques du monde académique, l'autre répondant à des préoccupations davantage sociétales en s'éloignant du trait conservateur de ce monde académique. Nous proposons ainsi d'élargir la typologie des organisations qui tendait à faire des communautés l'alternative dominante aux formes hiérarchiques. Aux côtés des communautés académiques, les collectifs peuvent prendre leur place.

Toutefois, notre analyse reste limitée à un petit nombre de cas. Il serait souhaitable de travailler sur un plus large panel intégrant des communs développés dans différentes aires culturelles. Il serait également souhaitable de disposer d'une diversité de disciplines scientifiques et de contextualiser les pratiques à l'aide de variables telles que la maturité de la discipline au sein du paysage scientifique national. 
Il serait bienvenu d'approfondir l'intérêt porté aux formes de gouvernance des ressources en travaillant de manière pluridisciplinaire : revisiter la sociologie des sciences afin d'interroger les différentes modalités de regroupement des ressources, et s'inspirer des recherches développées dans l'économie du numérique pour diversifier les dimensions d'analyse.

Pour notre part, l'analyse linguistique participe de cet enrichissement des études. L'analyse des conventions et des imaginaires linguistiques laisse entrevoir des perspectives fécondes.

Le langage n'est pas simple moyen d'expression, il est ressource de symbolisation afin d'ordonner les organisations et en particulier les communs et ainsi structurer leurs espaces de communication (Auroux, 1994).

\section{RÉFÉRENCES}

AKRICH, M., CALLON, M., LATOUR, B. (dir.) (2006), Sociologie de la traduction : textes fondateurs, Paris, Presses des Mines de Paris.

AMIN, A., ROBERTS, J. (2008), Community, Economic Creativity and Organization, Oxford, Oxford University Press.

AUROUX, S. (1994), La révolution technologique de la grammatisation, Paris, Éditions Mardaga.

BARBERIO, V., MONTI, A. (2014), Réseaux sémantiques et légitimé du discours organisationnel : une illustration empirique, Communiquer, 12, 7-25.

BAKHTINE, M. (1993), Toward a Philosophy of the Act, Austin, ed., M. Holquist, Texas University Press, traduction française (2003) Pour une philosophie de l'acte, Lausanne, L'Âge d'Homme.

BOURDIEU, P. (1975), La spécificité du champ scientifique et les conditions sociales du progrès de la raison, Sociologie et sociétés, 7(1), 91-118.

BROWN, J. S., DUGUID, P. (1991), Organizational Learning and Communities-OfPractice: Toward a Unified View of Working, Learning and Innovation, Organization Science, 2(1), 40-57.

CLEGG, S., RHODES, C., KORNBERGER, M. (2007), Desperately Seeking Legitimacy: Organizational Identity and Emerging Industries, Organization Studies, 28, 495-513.

CLOT, Y., BÉGUIN, P. (2004), Laction située dans le développement de l'activité, Activités, http://journals.openedition.org/activites/1237, mis en ligne le 1er octobre 2004.

COWAN, R., DAVID, P., FORAY, D. (2000), The Explicit Economics of Knowledge Codification, Industrial and Corporate Change, 9(2), 211-253.

COHENDET, P., LLERENA, P. (2003), Routines and Incentives: The Role of Communities in the Firm, Industrial and Corporate Change, 12(2), 271-297.

COHENDET, P., GRANDADAM, D., SIMON, L. (2008), Réseaux, communautés et projets dans les processus créatifs, Management International, 13(1), 29-43.

CORIAT, B. (ed.) (2015), Le retour des communs, Paris, Les Liens qui libèrent. 
CZARNIAWSKA, B. (1997), Narrating the Organization, Dramas of Institutional Identity, Chicago, IL, The University of Chicago Press.

DANG NGUYEN, G., DEJEAN, S., JULLIEN, N. (2017), Do Open Online Projects Create Social Norms?, Journal of Institutional Economics, 14(1), 45-70.

DAVID, P. A., FORAY, D. (2002), An Introduction to the Economy of the Knowledge Society, International Social Science Journal, 54(171), 9-23.

FERRY, J. M. (1991), Les puissances de l'expérience, tome I, Paris, Éditions du Cerf.

FERRY, J. M. (2004), Les grammaires de l'intelligence, Paris, Éditions du Cerf.

FIOL, M. (2002), Capitalizing on Paradox: The Role of Language in Transforming Organizational Identities, Organization Science, 13, 653-666.

FLECK, L. (1934), Genèse et développement d'un fait scientifique, Paris, Les Belles Lettres.

FORAY, D. (2004), The Economics of Knowledge, Cambridge MA, MIT Press.

GIRIN, J. (1990), Problèmes du langage dans les organisations, in Chanlat et al., L'individu dans l'organisation : les dimensions oubliées, Laval, Presses Universitaires de Laval, Québec, ESKA, Paris, 37-77.

GRANJOU, C., PEERBAYE, A. (2011), Sciences et collectifs. Introduction, Revue de Sciences Sociales, Terrains et Travaux, 18, 5-18.

GIBBONS, M., LIMOGES, C., NOWOTNY, H., SCHWARTZMAN, S., SCOTT, P., TROW, M. (1994), The New Production of Knowledge: The Dynamics of Science and Research in Contemporary Societies, London, Thousand Oaks.

HAAS, P. M. (1992), Epistemic Communities and International Policy Coordination, International Organization, 46(1), 1-35.

HAGSTROM, W. O. (1965), The Scientific Community, New York, Basic Books.

HAJEK, I. (2011), Sociologie, cognition et langage : une méthodologie d'observation, Langage et Société, 135(1), 67-85.

HJELMSLEV, L. (1942), Langue et parole, Cahiers Ferdinand de Saussure, 2, 29-44.

HOLZNER, B., MARX, J. (1979), Knowledge Application: The Knowledge System in Society, Boston, Allyn and Bacon.

HOUDEBINE, A.M. (1985), Pour une linguistique synchronique dynamique, La linguistique, 21, 7-36.

HOUDEBINE-GRAVAUD, A. M. (1993), De l'imaginaire des locuteurs et de la dynamique linguistique : aspects théoriques et méthodologiques, in Francard, M. (dir.), L'insécurité linguistique dans les communautés francophones périphériques, Louvain-laNeuve, Peeters/Institut de linguistique, 31-40.

HOUDEBINE-GRAVAUD, A. M. (2013), L'imaginaire linguistique entre idéal de langue et langue idéale. Sa modélisation, son application, son développement en imaginaire culturel via la sémiologie des indices, Proceedings of the 10th International Conference of the Faculty of Letters of Pitesti, Juin, 9-19, Roumanie.

KNORR-CETINA, K. (1982), Scientific Communities or Transepistemic Arenas of Research?, Social Studies of Science, 12, 101-30.

KNORR-CETINA, K. (1999), Epistemic Cultures. The Cultures of Knowledge Societies, Cambridge, Harvard University Press. 
KUHN, T. S. (1962), The Structure of Scientific Revolutions, Chicago, University of Chicago Press.

LATOUR, B. (2005), Transmettre la Syphilis - Partager l'objectivité, in Fleck, L, Genèse et développement d'un fait scientifique, Paris, Les Belles Lettres.

LIVET, P. (1994), La communauté virtuelle. Action et communication, Combas, Éditions de l'Éclat.

MARTINET, A. (1969), Peut-on dire d'une langue qu'elle est belle, Le français sans fard, Paris, Presses Universitaires de France, 46-61.

MERTON, R. K. (1973), The Sociology of Science: Theoretical and Empirical Investigations, Chicago, University of Chicago Press.

MEYER, M., MOLYNEUX-HODGSON, S. (2011), Communautés épistémiques : une notion utile pour théoriser les collectifs en sciences ?, Terrains $\mathcal{E}$ Travaux, 18(1), 141154.

OSTROM, E. (1990), Governing the Commons: The Evolution of Institutions for Collective Action, Cambridge, Cambridge University Press.

PHILLIPS, N., OSWICK, C. (2012), Organizational Discourse: Domains, Debates, and Directions, The Academy of Management Annals, 6(1), 435-481.

REMYSEN, W. (2011), Lapplication du modèle de l'Imaginaire linguistique à des corpus écrits : le cas des chroniques de langage dans la presse québécoise, Langage et société, 135(1), 47-65.

SHINN, T. (2002), Nouvelle production du savoir et triple hélice. Tendances du prêt-àpenser les sciences, Actes de la recherche en sciences sociales, 1, 141-142, 21-30.

SOULIER, E. (2017), La pratique : un phénomène, une perspective ou une ontologie ? Quelques clés de lecture pour identifier, délimiter et caractériser une pratique, in Soulier, E., Audran, J. (dir.), Communautés de pratique et management de la formation, Montbéliard, Presses de l'Université de Belfort Montbéliard.

STALLMAN, R. (2002), Free Software, Free Society: Selected Essays of Richard M. Stallman, GNU Press.

SUCHMAN, L. (1987), Plans and Situated Actions: The Problem of Human-Machine Communication, Cambridge, Cambridge University Press.

WEICK, K. (1995), Sensemaking in Organizations, Thousand Oaks, CA, Sage Publications.

WENGER, E. (1998), Communities of Practice: Learning, Meaning and Identity, New York, Cambridge University Press. 